\title{
Experiment-friendly formulation of quantum backflow
}

\author{
Marek Miller ${ }^{1,2}$, Woo Chee Yuan ${ }^{1}$, Rainer Dumke ${ }^{1,3}$, and Tomasz Paterek ${ }^{1,4,5}$ \\ ${ }^{1}$ School of Physical and Mathematical Sciences, Nanyang Technological University, Singapore \\ ${ }^{2}$ Centre of New Technologies, University of Warsaw, Poland \\ ${ }^{3}$ Centre for Quantum Technologies, National University of Singapore, Singapore \\ ${ }^{4}$ MajuLab, International Joint Research Unit UMI 3654, CNRS, Universite Cote d'Azur, Sorbonne Universite, National University of \\ Singapore, Nanyang Technological University, Singapore \\ ${ }^{5}$ Institute of Theoretical Physics and Astrophysics, Faculty of Mathematics, Physics and Informatics, University of Gdańsk, Poland
}

Quantum backflow is usually understood as a quantum interference phenomenon where probability current of a quantum particle points in the opposite direction to particle's momentum. Here, we quantify the amount of quantum backflow for arbitrary momentum distributions, paving the way towards its experimental verification. We give examples of backflow in gravitational and harmonic potential, and discuss experimental procedures required for the demonstration using atomic gravimeters. Such an experiment would show that the probability of finding a free falling particle above initial level could grow for suitably prepared quantum state with most momentum downwards.

\section{Introduction}

A wave function of a quantum particle has physically observable characteristics that can be local or global. The probability of finding the particle in a specific region of space or the probability current are examples of local characteristics, which can be determined given access to only small part of the wave function. In contradistinction, the momentum is a property of the entire wave function, e.g. requires the determination of the de Broglie wavelength. Already at this level of generality, it is clear that the probability current and the momentum of a quantum particle may behave very differently.

Quantum backflow (QB) is an interference effect that arises from this observation. In order to understand the statement better, and to simplify the subsequent analysis, let us suppose that a particle in the one-dimensional space interacts with a potential that depends only on the particle's position. Intuitively, we may think that if the momentum distribution concentrates within the positive half-line, the probability current, too, will be non-negative. It turns out that

Marek Miller:

m.miller@cent.uw.edu.pl

Woo Chee Yuan:

P160031@e.ntu.edu.sg this is not the case for a suitably chosen quantum state. In a sense, the probability flows 'backwards'. Hence the term "quantum backflow".

QB was first studied by Allcock in his work on the arrival time in quantum mechanics [2] (see also Kijowski [17] for an early discussion of QB). Later, Bracken and Melloy [7] gave a detailed analysis of QB as an eigenvalue problem. The analysis was rigorously phrased in the mathematical language of integral operators on separable Hilbert space in [24]. In the same paper, a numerical approximation of the optimal QB state was given. See also $[16,28]$ for intresting case studies. QB in systems interacting with linear potential was studied in [21]. Recently, there have been attempts at analysing QB in the relativistic setting $[20,25,3]$, in the setting of quantum particle decay $[9,13]$, as well as the attempts at describing quantum backflow in dissipative [22] and many-particle systems [4]. The phenomenon was extended into phase space in Ref. [14] and systems suitable for observing QB in an experiment were discussed in Refs. [27, 15].

Among others, Palmero et al. [23] proposed an experimental scheme that "could lead to the observation of quantum backflow" in ${ }^{7} \mathrm{Li}$ Bose-Einstein condensate. To the best of our knowledge, however, QB has not yet been confirmed experimentally. Note that in a recent experimental work by Eliezer et al. [10], "optical backflow" in transverse momentum of a beam of light was reported. The present study, however, focuses on quantum backflow of individual non-relativistic quantum particles. We extend the customary definition of QB to states with non-zero probability of measuring negative momentum. Hence, our approach should be applicable to realistic experimental situations. We study systems exhibiting QB in gravitational field near the surface of the Earth, as well as interacting with harmonic potential. We also comment on possible experimental verification of $\mathrm{QB}$ using atomic gravimeters.

\section{Quantum backflow}

Our definition of quantum backflow conveys how necessary conditions for the probability current that fol- 
low from the classical equations of motion are no longer satisfied in the quantum regime.

Let us focus on the system of a lone particle in the one-dimensional space. We set the vertical direction with the $x$ axis pointing downwards. Suppose the particle interacts with arbitrary potential $V(x)$. We examine its dynamics from classical and quantum points of view with initial conditions as similar as possible. The quantum system at time $t$ is fully described by its wave function $\psi_{t}(x)$. The classical model requires simultaneous knowledge of position and momentum, whose precise estimation is famously forbidden by the uncertainty principle. We therefore propose an operational approach in which distribution of position and momentum is estimated with finite precision, and given by the probability density function

$$
f_{t}(x, p)=|\langle\phi|W(x, p)| \psi\rangle|^{2},
$$

where $W(x, p)$ is the Wigner-Moyal transform of $\psi$ and $\phi$ (see e.g. Eq. (6.63) in [8]):

$$
\begin{aligned}
& \langle\phi|W(x, p)| \psi\rangle= \\
& \quad=\frac{1}{2 \pi \hbar} \int_{-\infty}^{\infty} e^{-\frac{i}{\hbar} p y} \phi^{*}\left(y-\frac{x}{2}\right) \psi\left(y+\frac{x}{2}\right) d y .
\end{aligned}
$$

The function $\phi$ represents the finite precision of the measurement apparatus and, for example, can be a Gaussian distribution, centred at zero, with finite width $\sigma_{\phi}$. The marginals of $f_{t}(x, p)$ agree with densities of position and momentum, derived from $\psi_{t}(x)$, "up to $\sigma_{\phi}^{2}$ ", i.e.:

$$
\begin{aligned}
& P_{t}\left(x=x_{0}\right)=\int_{-\infty}^{\infty} f_{t}\left(x_{0}, p\right) d p=\left(|\psi|^{2} *|\phi|^{2}\right)\left(x_{0}\right), \\
& P_{t}\left(p=p_{0}\right)=\int_{-\infty}^{\infty} f_{t}\left(x, p_{0}\right) d x=\left(|\tilde{\psi}|^{2} *|\tilde{\phi}|^{2}\right)\left(p_{0}\right),
\end{aligned}
$$

where $\tilde{\psi}(p)$ and $\tilde{\phi}(p)$ are Fourier transforms of $\psi$ and $\phi$, respectively, and the symbol ' $*$ ' stands for convolution.

In other words, the family of operators $W(x, p)^{\dagger}|\phi\rangle\langle\phi| W(x, p)$, defined on the phase space $(x, p) \in \mathbb{R}^{2}$, is a positive operator-valued measure that allows experimental estimation of the joint probability distribution $f_{t}(x, p)$ of position and momentum in the state $\psi$, with a finite precision given by a square-integrable function $\phi$.

Let us now derive probability currents in the quantum and classical models corresponding to the rate of change of probability of finding the particle above the level $x=a$. In the quantum case, this is a textbook exercise leading to the familiar formula

$$
\begin{aligned}
j_{t}(a)=-\frac{d}{d t} P_{t} & (x \leq a)= \\
= & \frac{\hbar}{m} \mathfrak{I m}\left(\left.\psi_{t}^{*}(a) \frac{d}{d x} \psi_{t}(x)\right|_{x=a}\right),
\end{aligned}
$$

where $\mathfrak{I m}$ stands for the imaginary part of a complex number.

Let us fix $t$ for now and consider a putative classical system, with the distribution of position and momentum given by $f_{t}(x, p)$. The distribution evolves for a short time $\tau, t \leq \tau \leq t+\Delta t$ according to the Hamilton equations of motion: $\dot{x}(\tau)=p / m, \quad \dot{p}(\tau)=-\frac{d V}{d x}$. This implies that, unlike in the quantum case, the probability current of the classical system,

$$
\left(j_{\mathrm{cl}}\right)_{t}(a)=-\left.\frac{d}{d \tau} P_{\mathrm{cl}}(x(\tau) \leq a)\right|_{\tau=t},
$$

must be bounded from below:

$$
\left(j_{\mathrm{cl}}\right)_{t}(a) \geq \frac{1}{m} \int_{-\infty}^{0} p f_{t}(a, p) d p
$$

(see Appendix for the detailed proof). We can say that the probability of finding the classical particle above the line $x=a$ cannot grow faster than a certain quantity derived from the distribution of only negative momenta. In particular, if $f_{t}(x, p)=0$ for $p<0$, we get that $\left(j_{\mathrm{cl}}\right)_{t}(a) \geq 0$, i.e. the direction of the momentum and the probability current coincide. In this particular case, it leads to the usual definition of QB given by the following statements about a wave function of a quantum system in one-dimensional space [6]: a) $\tilde{\psi}_{t}$ contains only positive momenta; b) there exists $a \in \mathbb{R}$, for which $j_{t}(a)<0$. To facilitate the analysis of QB for arbitrary states, we say that QB is a situation where the inequality (7) no longer holds.

Definition 1. The quantum backflow takes place at point $x=a$ and at time $t$, if

$$
j_{t}(a)<\frac{1}{m} \int_{-\infty}^{0} p f_{t}(a, p) d p,
$$

where the probability current $j_{t}(a)$ is given by Eq. (5) and the function $f_{t}(x, p)$ by Eq. (1).

Note that by taking a formal limit of the precision function converging to the Dirac delta distribution in the momentum representation, $\tilde{\phi}(p) \rightarrow \delta(p)$, we can see from Eq. (2) that $f_{t}(x, p) \rightarrow|\tilde{\psi}(p)|^{2}$, at any point $x$. Hence, in this limiting case, and for a wave function such that $\tilde{\psi}(p)=0$ for $p<0$, the inequality (8) reduces to the standard definition of $\mathrm{QB}$, requiring only $j_{t}(a)<0$.

It is also worth mentioning that Eq. (8) is a condition independent of whether the system undergoes quantum scattering. For example, it is easy to see that an initial superposition of plain waves,

$$
\psi(x)=A e^{i k x}+B e^{-i k x}, \quad x<0,
$$

scattered back on a potential step, does not exhibit quantum backflow. Unlike scattering, quantum backflow is an instantaneous property of the particle's quantum state, namely its probability current exceeding a classical bound, regardless of the underlying dynamics. 

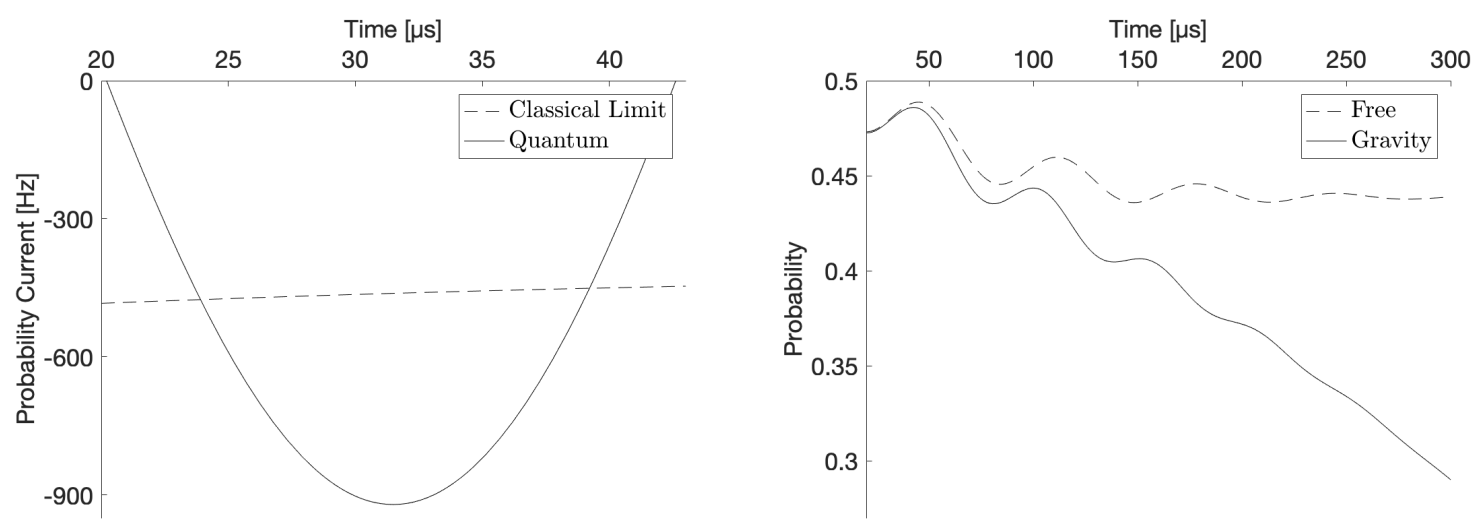

Figure 1: Quantum backflow in gravity. The initial state is a superposition of two Gaussian wave functions with amplitudes given in (12), describing Rubidium atom. Left panel: The dashed line gives the lower bound on the classical probability current. The solid line is the quantum probability current. QB takes place in the interval when the solid line is below the dashed line, see Eq. (8). Right panel: As a consequence, the probability $P(x<0)$ of finding the particle above the initial level of $x=0$ increases despite small contributions from negative momenta. Solid line gives the probability in the presence of the gravitational field. For comparison, the dashed line represents the free particle.

\section{Examples}

We give two concrete examples of $\mathrm{QB}$ according to Definition 1. Both involve superposition of Gaussian states and therefore naturally contain contributions from negative momenta. The first example is a particle in a linear potential as an approximation of the Newtonian gravity close to the Earth's surface. The second example considers quadratic (harmonic) potential.

\subsection{Gravitational potential}

Suppose the particle interacts with the potential $V(x)=-m g x$, for $g \geq 0$. Recall that by our convention, the direction of the $x$ axis and the direction of the gravitational force coincide. We choose the initial level $x=0$ above the surface. Of course, by putting $g=0$, we also cover the case of a free particle on the real line.

Consider the initial wave function, at time $t=0$, being a superposition of two Gaussian states centred at $x=0$, with the same spread $\sigma$ but different mean momenta. If the corresponding quantum particle is free, the wave function at time $t$ reads:

$$
\begin{aligned}
& \psi_{\text {free }}(x, t)=\sum_{n=1}^{2} \frac{B_{n}}{\sqrt{4 \sigma^{2}+2 i \frac{h}{m} t}} \times \\
& \times \exp \left(\frac{i}{\hbar} p_{n}\left(x-\frac{p_{n}}{2 m} t\right)-\frac{\left(x-\frac{p_{n}}{m} t\right)^{2}}{4 \sigma^{2}+2 i \frac{h}{m} t}\right),
\end{aligned}
$$

where $p_{1}, p_{2}$ are the mean values of momentum for each branch of the superposition and $B_{1}, B_{2}$ are the corresponding probability amplitudes. In the presence of the linear potential $V(x)$, the wave function accelerates as a result of the gravitational force, and at time $t$ it takes the form [26]:

$$
\psi(x, t)=e^{-\frac{i}{\hbar}\left(-m g t x+\frac{1}{6} m g^{2} t^{3}\right)} \psi_{\text {free }}\left(x-\frac{1}{2} g t^{2}, t\right) .
$$

Let us now choose realistic values of the parameters (see next section for further discussion of a possible experiment). E.g., let us take the Rubidium atom of mass $m \approx 1.4 \times 10^{-25} \mathrm{~kg}$, described by the wave function with $\sigma=1 \mu \mathrm{m}$. The mean momenta are set to $p_{1}=0$ and $p_{2}=2 \hbar k$, where the wave number $k=2 \pi / \lambda$ with $\lambda=780 \mathrm{~nm}$. The following amplitudes

$$
B_{1} \approx 1.18 \times 10^{-3}, B_{2} \approx 4.42 \times 10^{-4},
$$

numerically optimise the effect of $\mathrm{QB}$. The precision function is set to be a Gaussian with standard deviation $\sigma_{\phi}=0.1 \mu \mathrm{m}$.

In Fig. 1, we show the probability current and the overall probability of finding the particle above the initial line $x=0$ as functions of time. We set $g=9.8 \mathrm{~m} \cdot \mathrm{s}^{-2}$. It is clear that for $t$ such that approximately: $23.7 \mu \mathrm{s} \leq t \leq 39 \mu$ s, the probability current satisfies the inequality (8), i.e. QB takes place. During that time, the numerical value of the integral $\int_{-\infty}^{0}|\tilde{\psi}(p)|^{2} d p$, i.e. the contribution of "negative momenta" to the backflow state, ranges from 0.23 to 0.13 . Here, our approach allows us to separate the contribution of the negative momenta from the genuine quantum backflow. Despite the particle's free fall, the quantum probability of finding the particle above the initial level unmistakably increases.

\subsection{Harmonic potential}

Our second example is a particle in quadratic potential, due to its wide applicability. We again consider $\mathrm{Rb}$ atom, but this time in a harmonic trap with frequency $\nu=10 \mathrm{kHz}$ [5]. It is generally known that 


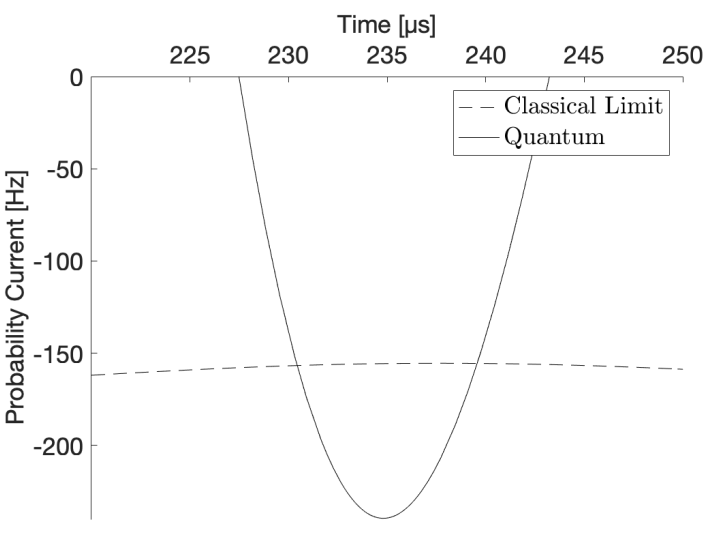

Figure 2: Quantum backflow in a harmonic trap. $\mathrm{Rb}$ atom in the initial superposition of coherent states with parameters given in the main text gives rise to the quantum probability current (solid line) which is below the classical lower bound (dashed line). This signifies QB for approximately $(230,239) \mu \mathrm{s}$. Probability current was evaluated at $x=\sqrt{\frac{2 \hbar}{m \omega}} \cos (0.55 \pi)$.

coherent states of a quantum harmonic oscillator take the form of a Gaussian packet in position representation [18]. Hence, we take as an initial state the superposition of two coherent states $|\psi\rangle=a|\alpha\rangle+a|\beta\rangle$. Numerical optimisation of $\mathrm{QB}$ leads to the following parameters: $a \approx 0.73, \alpha=e^{i(0.9 \pi-\omega t)}, \beta=9 e^{i(0.55 \pi-\omega t)}$, where $\omega=2 \pi \nu$. Fig. 2 shows the corresponding classical bound on the probability current and the quantum prediction for $\mathrm{QB}$.

\section{Experimental proposal}

The approach proposed here is based on the measurement of probability current and generalised joint measurement (with finite precision) of position and momentum. The latter determines the right-hand side of Eq. (8) and the former its left-hand side. Probability current is a convenient theoretical quantity in this problem because in the derivation of (8) Hamilton's equations reduce the classical bound to the integral over momentum only. In more practical terms, the measurement of the probability current requires estimation of particle's position at two nearby times and division by the corresponding time interval. This can be simplified to only one estimation of particle's position by using the bound of Eq. (18) in the Appendix.

The essence of the present proposal is to establish the initial joint probability distribution of position and momentum that is used to compute the classical bound. Since this might be experimentally quite demanding, we offer one more route to the verification of QB, based on independent measurements of initial position and momentum distributions. Such measurements do not give any information about possible position-momentum correlations. Accordingly, we propose to consider all joint distributions $f_{t}(x, p)$ compatible with these marginals and take the worst case upper bound in Eq. (18).

Finally, a concrete setup capable of measuring QB could be constructed using atomic gravimeters. High precision estimation of position distribution has been realised with electron [12] as well as optical microscopy $[1,19]$ of the atomic cloud; and momentum distribution can be established from the time-of-flight measurements [11]. Depending on the way the atoms are trapped these setups offer initial width of the wave function in the range from micrometers to millimetres. We have verified numerically that QB is present for all these spreads with the order of magnitude of the bound and the quantum probability current scaling like inverse square of the width.

\section{Conclusions}

We presented an analysis of QB for states with admissible negative momentum. Unlike the standard definition of QB, Def. 1 is applicable not only to wave functions with positive support in momentum representation. Broadly speaking, our idea is to compare the classical evolution of the joint probability distribution of position and momentum (known with finite precision) with the quantum dynamics. The classical system would evolve for an infinitesimally short time according to Hamilton's equations of motion, starting with the same initial conditions. We defined $\mathrm{QB}$ as a situation when the probability current exceeds what might be possible for its classical counterpart.

In particular, we showed that a relatively easy to prepare superposition of two Gaussian packets exhibits QB during free-fall in the uniform gravitational field near the Earth's surface. Of course, the Ehrenfest theorem guarantees that the average position of the quantum particle follows the classical trajectory. Nevertheless, the probability of locating the particle above the initial level displays 'antigravitational' quantum backflow.

\section{Acknowledgments}

We would like to warmly thank Referees for their insightful comments and questions.

This work is supported by the Polish National Agency for Academic Exchange NAWA Project No. PPN/PPO/2018/1/00007/U/00001. 


\section{A Appendix}

Here, we give a proof of the inequality (7).

Once again, we consider the distribution of position and momentum given by $f_{t}(x, p)$ that evolves classically for a brief time $\tau, t \leq \tau \leq t+\Delta t$. If our system were initially at point $x$, then $x(\tau)=x+\frac{p}{m}(\tau-t)$. The probability $P(x(\tau) \leq a)$ of finding the particle in the region "above" the line $x=a$ would be

$$
\begin{aligned}
P(x(\tau) \leq a) & =P\left(x \leq a-\frac{p}{m}(\tau-t)\right) \\
& =\int_{-\infty}^{a-\frac{p}{m}(\tau-t)} \int_{-\infty}^{\infty} f_{t}(x, p) d p d x \\
& =\int_{-\infty}^{a} \int_{-\infty}^{\infty} f_{t}\left(x-\frac{p}{m}(\tau-t), p\right) d p d x .
\end{aligned}
$$

Now, the probability current of the classical system,

$$
\left(j_{\mathrm{cl}}\right)_{t}(a)=-\left.\frac{d}{d \tau} P(x(\tau) \leq a)\right|_{\tau=t},
$$

can be expressed in terms of the probability density function $f_{t}(x, p)$ :

$$
\begin{aligned}
\left(j_{\mathrm{cl}}\right)_{t}(a) & =-\left.\frac{d}{d \tau} \int_{-\infty}^{a} \int_{-\infty}^{\infty} f_{t}\left(x-\frac{p}{m}(\tau-t), p\right) d p d x\right|_{\tau=t} \\
& =\int_{-\infty}^{a} \int_{-\infty}^{\infty} \frac{p}{m} \frac{\partial}{\partial x} f_{t}(x, p) d p d x \\
& =\frac{1}{m} \int_{-\infty}^{\infty} p f_{t}(a, p) d p,
\end{aligned}
$$

where the last equality is a consequence of the fact that $\lim _{x \rightarrow \pm \infty} f_{t}(x, p)=0$. This immediately yields the inequality:

$$
\left(j_{\mathrm{cl}}\right)_{t}(a) \geq \frac{1}{m} \int_{-\infty}^{0} p f_{t}(a, p) d p .
$$

From a practical point of view, in order to save on measurements, it would be more desirable to derive the classical upper bound for the probability of finding the particle above the initial level instead of the lower bound on the probability current. For this we use Eq. (13) and note the following inequality

$$
\begin{aligned}
P(x(\tau) \leq a) \leq & \int_{-\infty}^{a-\frac{p}{m}(\tau-t)} \int_{-\infty}^{0} f_{t}(x, p) d p d x \\
& +\int_{-\infty}^{a} \int_{0}^{\infty} f_{t}(x, p) d p d x
\end{aligned}
$$

which is simply a consequence of non-negativity of $f_{t}(x, p)$. Hence,

$$
\begin{aligned}
P(x(\tau) \leq a) & \leq P(x \leq a) \\
& +\int_{a}^{a-\frac{p}{m}(\tau-t)} \int_{-\infty}^{0} f_{t}(x, p) d p d x .
\end{aligned}
$$

\section{References}

[1] A. Alberti et al. "Super-resolution microscopy of single atoms in optical lattices". In: New J. Phys. 18 (2016), p. 053010. DOI: https://doi.org/10.1088/13672630/18/5/053010.

[2] Go.R. Allcock. "The time of arrival in quantum mechanics I. Formal considerations". In: Annals of Physics 53.2 (1969), pp. 253-285. DOI: https://doi.org/10.1016/0003-4916(69)90251-6.

[3] J. Ashfaque, J. Lynch, and P. Strange. "Relativistic quantum backflow". In: Physica Scripta (2019). DOI: https://doi.org/10.1088/14024896/ab265c.

[4] M. Barbier. "Quantum backflow for many-particle systems". In: Physical Review A 102 (2020), p. 023334. DOI: https://10.1103/PhysRevA.102.023334.

[5] T.J. Beams, G. Peach, and I.B. Whittingham. "Ultracold atomic collisions in tight harmonic traps: perturbation theory, ionization losses and application to metastable helium atoms". In: Journal of Physics B: Atomic, Molecular and Optical Physics 37.22 (2004), pp. 45614570. DOI: https://doi.org/10.1088/09534075/37/22/014.

[6] H. Bostelmann, D. Cadamuro, and Ga.f Lechner. "Quantum backflow and scattering". In: Physical Review A 96.1 (2017), p. 012112. DOI: https://doi.org/10.1103/PhysRevA.96.012112.

[7] A.J. Bracken and G.F. Melloy. "Probability backflow and a new dimensionless quantum number". In: Journal of Physics A: Mathematical and General 27.6 (1994), p. 2197. DOI: https://doi.org/10.1088/0305-4470/27/6/040.

[8] M.A. De Gosson. Symplectic geometry and quantum mechanics. Vol. 166. Springer Science \& Business Media, 2006. DOI: https://doi.org/10.1007/3-7643-7575-2.

[9] W. van Dijk and F.M. Toyama. "Decay of a quasistable quantum system and quantum backflow". In: Physical Review A 100.5 (2019), p. 052101. DOI: https://doi.org/10.1103/PhysRevA.100.052101. 
[10] Y. Eliezer, T. Zacharias, and A. Bahabad. "Observation of Optical Backflow". In: Optica 7 (2020), p. 72. DOI: https://doi.org/10.1364/OPTICA.371494.

[11] J.R. Ensher et al. "Bose-Einstein Condensation in a Dilute Gas: Measurement of Energy and Ground-State Occupation". In: Phys. Rev. lett. 77 (1996), p. 4984. DOI: https://doi.org/10.1103/PhysRevLett.77.4984.

[12] T. Gericke et al. "High-resolution scanning electron microscopy of an ultracold quantum gas". In: Nat. Phys. 4 (2008), 949-953. DOI: https://doi.org/10.1038/nphys1102.

[13] A. Goussev. "Equivalence between quantum backflow and classically forbidden probability flow in a diffraction-in-time problem". In: Physical Review A 99.4 (2019), p. 043626. DOI: https://doi.org/10.1103/PhysRevA.99.043626.

[14] A. Goussev. "Probability backflow for correlated quantum states". In: Physical Review Research 2 (2020), p. 033206. DOI: https://doi.org/10.1103/PhysRevResearch.2.033206.

[15] A. Goussev. "Quantum backflow in a ring". In: arXiv preprint arXiv:2008.08022 (2020).

[16] J.J. Halliwell et al. "Quantum backflow states from eigenstates of the regularized current operator". In: Journal of Physics A: Mathematical and Theoretical 46.47 (2013), p. 475303. DOI: https://doi.org/10.1088/17518113/46/47/475303.

[17] J. Kijowski. "On the time operator in quantum mechanics and the Heisenberg uncertainty relation for energy and time". In: Reports on Mathematical Physics 6.3 (1974), pp. 361386. DOI: https://doi.org/10.1016/S00344877(74)80004-2.

[18] J.R. Klauder and B.-S. Skagerstam. Coherent States: Applications in Physics and Mathematical Physics. World Scientific, Singapore, 1985. DOI: https://doi.org/10.1142/9789814415118_0007.

[19] M. McDonald et al. "Superresolution Microscopy of Cold Atoms in an Optical Lattice". In: Phys. Rev. X 9 (2019), p. 021001. DOI: https://doi.org/10.1103/PhysRevX.9.021001.

[20] G.F. Melloy and A.J. Bracken. "Probability backflow for a Dirac particle". In: Foundations of Physics 28.3 (1998), pp. 505-514. DOI: https://doi.org/10.1023/A:1018724313788.

[21] G.F. Melloy and A.J. Bracken. "The velocity of probability transport in quantum mechanics". In: Annals of Physics 7.7-8 (1998), pp. 726731. DOI: https://doi.org/10.1002/(SICI)15213889(199812)7:7/8\%3C726::AIDANDP726\%3E3.0.CO;2-P.
[22] S..V Mousavi and S. Miret-Artés. "Dissipative quantum backflow". In: The European Physical Journal Plus 135.3 (2020), pp. 1-18. DOI: https://doi.org/10.1140/epjp/s13360-02000336-5.

[23] M. Palmero et al. "Detecting quantum backflow by the density of a BoseEinstein condensate". In: Physical Review A 87.5 (2013), p. 053618. DOI: https://doi.org/10.1103/PhysRevA.87.053618.

[24] M. Penz et al. "A new approach to quantum backflow". In: Journal of Physics A: Mathematical and General 39.2 (2005), p. 423. DOI: https://doi.org/10.1088/0305-4470/39/2/012.

[25] H.-Y. Su and J.-L. Chen. "Quantum backflow in solutions to the Dirac equation of the spin-1 2 free particle". In: Modern Physics Letters A 33.32 (2018), p. 1850186. DOI: https://doi.org/10.1142/S0217732318501869.

[26] G. Vandegrift. "Accelerating wave packet solution to Schrödinger's equation". In: American Journal of Physics 68.6 (2000), pp. 576-577. DOI: https://doi.org/10.1119/1.19492.

[27] A.H. de Vasconcelos Jr. "Quantum backflow in the presence of a purely transmitting defect". In: arXiv preprint arXiv:2007.07393 (2020).

[28] J.M. Yearsley et al. "Analytical examples, measurement models, and classical limit of quantum backflow". In: Physical Review A 86.4 (2012), p. 042116. DOI: https://doi.org/10.1103/PhysRevA.86.042116. 